\title{
A THEOREM ON VALUATION RINGS AND ITS APPLICATIONS
}

\author{
MASAYOSHI NAGATA"
}

To Professor K. Noshiro on his 60 th birthday

In the present paper, we first prove the following

Theorem 1. Let $K$ be a field, $x$ a transcendental element over $K$, and $V^{*} a$ valuation ring of $K(x)$. Set $V=V^{*} \cap K$. Denote by $\mathfrak{p}^{*}$ and $\mathfrak{p}$ the maximal ideals of $V^{*}$ and $V$ respectively. If (i) $V^{*} / p^{*}$ is not algebraic over $V / \mathfrak{p}$ and (ii) the value group of $V^{*}$ is isomorphic to $\mathrm{Z}^{n}$ ( $\mathrm{Z}=$ the module of rational integers), i.e., $V^{*}$ is of rank $n$ and discrete in the generalized sense, ${ }^{2)}$ then $V^{*} / p^{*}$ is a simple transcendental extension of a finite algebraic extension of $V / p$.

Then we show some applications of this theorem to the theory of fields. At the end of this paper, we shall discuss the theorem above without assuming (ii).

Besides usual terminology on rings and fields, we make the following definitions : (1) a field $L$ is said to be ruled over its subfield $K$ if $L$ is a simple transcendental extension of its subfield containing $K,(2)$ a field $L$ is said to be anti-rational over its subfield $K$ if no finite algebraic extension of $L$ is ruled over $K$ and (3) a field $L$ is said to be quasi-rational over its subfield $K$ if every subfield of $L$ which contains $K$ properly is not anti-rational over $K$.

The writer likes to express his thanks to his friends in Harvard University and Purdue University, especially to Professor Zariski, for their discussion on these topics.

\section{Proof of Theorem 1}

We use induction argument on $n$. If $n=0$, then the assertion is obvious.

Received April 22, 1966.

1) The work was supported by NSF Grant GP-3512.

2) Under the presence of the condition (i), this (ii) is equivalent to that the value group of $V$ is isomorphic to $\mathbf{Z}^{n}$, as is easily seen from the fact that $V(y)=V[y] p V[v]$ is a valuation ring dominated by $V^{*}$ when $y \in V^{*}$ is transcendental modulo $p^{*}$ over $V / p$. 
Assume now that $n=1$. Since $K(x)=K\left(x^{-1}\right)$, we may assume that $x \in V^{*}$. Set $R=V[x]_{p^{*} \wedge V[x]}$. Since $V^{*}$ is a discrete valuation ring, so is $V$. If height $\left(\mathfrak{p}^{*} \cap V[x]\right)=1$, then $R$ is a valuation ring and $R=V^{*}$, hence $V^{*} / \mathfrak{p}^{*}=(V / \mathfrak{p})(x)$. Assume now that height $\left(p^{*} \cap V[x]\right) \neq 1$. Then $R$ is a regular local ring of Krull dimension 2 and the residue class field $R /\left(\mathfrak{p}^{*} \cap R\right)$ is a finite algebraic extension of $V / p$. Therefore this case is included in the following result of Abhyankar [1]: ${ }^{3)}$

Lemma 1. Let $K^{*}$ be a field with discrete ${ }^{4)}$ valuation ring $V^{*}$. If $R$ is a regular local ring of Krull dimension $r \geqq 2$ dominated by $V^{*}$ such that (i) the field of quotient of $R$ is $K^{*}$, (ii) trans.deg ${ }_{R / m} V^{*} / p^{*} \geq r-1, m$ and $\mathfrak{p}^{*}$ being the maximal ideals of $R$ and $V^{*}$ respectively, then $V^{*} / p^{*}$ is ruled over $R / \mathrm{m}$.

Proof. Starting with $R=R_{0}$, we consider the sequence of regular local rings $R_{i}$ such that each $R_{i}(i>0)$ is the quadratic dilatation of $R_{i-1}$ along $V^{*}$. Let $\mathfrak{p}_{i}$ be the maximal ideal of $R_{i}$. Since $V^{*}$ is discrete, $\cup R_{i}=V^{*}$. This shows that there is one $i$, say $j$, such that $V^{*} / \mathfrak{p}^{*}$ is algebraic over $R_{j} / \mathfrak{p}_{j}$; we choose the smallest $j$. At each step, trans. $\operatorname{deg}_{R_{i}} / \mathfrak{p}_{i} R_{i+1} / \mathfrak{p}_{i+1}+$ Krull-dim $R_{i+1} \leq$ Krull$\operatorname{dim} R_{i}$. Therefore $R$ may be replaced by $R_{j-1}$, hence we may assume that $j=1$. Then, denoting by $x_{1}, \ldots, x_{r}$ a regular system of parameters of $R$, we see that $x_{2} / x_{1}, \ldots, x_{r} / x_{1}$ are algebraically independent modulo $p^{*}$ over $R / p$. Therefore height $p_{1}=1$ and $R_{1}$ is a valuation ring. Thus $R_{1}=V^{*}$ and the proof of Lemma 1 is completed.

We go back to the proof of Theorem 1 . We consider the case $n>1$. Let $q^{*}$ be the prime ideal of height 1 in $V^{*}$ and set $\mathfrak{q}=q^{*} \cap V$. Compare $V^{*} / \mathfrak{q}^{*}$ and $V / \mathfrak{q}$. The residue class field of $V^{*} / q^{*}$ is not algebraic over that of $V / \mathfrak{q}$ by our assumption. This shows that $V^{*} / q^{*}$ is not algebraic over $V / q$ because these rings are valuation rings. Therefore the case $n=1$ can be applied to $V_{q^{\prime}}^{*}$ and $V_{\mathrm{q}}$, and we have $V_{q^{*}}^{*} / q^{*}=K^{\prime}(t)$ with a finite algebraic extension $K^{\prime}$ of $V_{\mathfrak{q}} / \mathrm{q}$. Let $V^{\prime}$ be $\left(V^{*} / q^{*}\right) \cap K^{\prime}$. Then $V^{\prime}$ is an extension of $V / q$ and the residue class field of $V^{\prime}$ is a finite algebraic extension of $V / p$. Therefore the induction assumption can be applied to $V^{*} / q^{*}$ and $V^{\prime}$, and we complete the proof of Theorem 1.

3) For the convenience of the readers, we shall give a complete proof.

4) This "discrete" may be understood to be the one of generalized sense. For, the presence of (ii) implies that $V^{*}$ must be of rank 1. 


\section{Lüroth theorem}

The original form of Lüroth theorem is as follows :

Let $K$ be a field. If $t$ is a transcendental element over $K$ and if $L$ is a subfield of $K(t)$ properly containing $K$, then $L$ is a simple transcendental extension of $K$.

As was proved by Igusa [2], ${ }^{5}$ this can be generalized as follows:

Lüroth Theorem. Assume that $t_{1}, \ldots, t_{n}$ are algebraically independent elements over a field $K$. If $L$ is a field between $K$ and $K\left(t_{1}, \ldots, t_{n}\right)$ such that trans. $\operatorname{deg}_{K} L=1$, then $L$ is a simple transcendental extension of $K$.

We show here that our Theorem 1 can be applied in order to derive this Lüroth theorem from its original form.

Lemma 2. Let $K$ and $K^{\prime}$ be fields of finite transcendence degree over a common field $k$. If an element $x$ is transcendental over both $K$ and $K^{\prime}$ and if $K(x)=K^{\prime}(x)$, then either $K \cong K^{\prime}$ or both $K$ and $K^{\prime}$ are ruled over $k^{6}{ }^{6}$

Proof. Consider the valuation ring $V^{*}=K[x]_{x K[x]}$ and set $V=K^{\prime} \cap V^{*}$. If $V$ contains $K^{\prime}$, then $V^{*}=K^{\prime}[x]_{x K^{\prime}[x]}$ and $K \cong V^{*} / x V^{*} \cong K^{\prime}$ in this case. If $V$ does not contain $K^{\prime}$, then the rank of $V$ is 1 , hence trans.deg $\operatorname{deg}_{k} V /\left(x V^{*} \cap V\right)$ $<$ trans. $\operatorname{deg}_{k} K^{\prime}=$ trans.deg $\operatorname{deg}_{k} K$. This shows that $V^{*} / x V^{+}$is not algebraic over $V /\left(x V^{*} \cap V\right)$. Therefore $K \cong V^{*} / x V^{*}$ is ruled. Symmetrically, we also have that $K^{\prime}$ is ruled. This completes the proof of Lemma 2.

Proof of Lüroth theorem. We use induction argument on $n$. If $n=1$, then it is the original form, and we assume that $n>1$. Then we may assume that $t_{1}$ is transcendental over $L$. Consider $K\left(t_{1}\right) \subset L\left(t_{1}\right) \subseteq K\left(t_{1}\right)\left(t_{2}, \ldots, t_{n}\right)$. Then by our induction assumption, we see that $L\left(t_{1}\right)=K\left(t_{1}, x\right)$ with a transcendental element $x$ over $K\left(t_{1}\right)$. Applying Lemma 2 to $L\left(t_{1}\right)=K(x)\left(t_{1}\right)$, we have either $L \cong K(x)$ or $L$ is ruled over $K$. Since $K$ is algebraically closed in $L$ (because so is in $\left.K\left(t_{1}, \ldots, t_{n}\right)\right)$, we have $L \cong K(x)$, completing the proof.

\section{Anti-rational fields}

ThEOREM 2. Let $k, K, L$ be fields and $x$ a transcendental element over $L$ such

5) In the classical case, this generalization was done by Weber [5].

6) Cf. Zariski problem which will be discussed in $\$ 5$. On the other hand, Samuel [3] proved that $K \cong K^{\prime}$ if furthermore (i) $k$ contains infinitely many elements and (ii) $K$ and $K^{\prime}$ are finitely generated over $k$. 
that (i) $k \subseteq K \subseteq L(x)$, (ii) $K$ is anti-rational over $k$ and (iii) $L$ is finitely generated over $k$. It follows then $K \subseteq L$.

Proof. Assume that $K \nsubseteq L$. Then $L \neq K(L) \subseteq L(x)$, and $L(x)$ is algebraic over $K(L)$. This shows that there is a transcendence base $t_{1}, \ldots, t_{n}$ of $L(x)$ over $K$ consisting only of elements of $L . \quad K\left(t_{1}, \ldots, t_{n}\right)$ has a valuation ring $V_{0}$ such that (i) $K \subseteq V_{0}$ and (ii) $V_{0}$ has prime ideals $p_{1} \supset p_{2} \supset \cdots \supset p_{n} \supset 0=p_{n+1}$ such that $t_{i} \in \mathfrak{p}_{i}, t_{i} \notin \mathfrak{p}_{i+1}$ for each $i=1, \ldots, n$ (hence, $\left.\mathfrak{p}_{i}=t_{i}\left(V_{0}\right)_{\mathfrak{p}_{i}}\right)$. The residue class field of $V_{0}$ is $K$. Let $V^{*}$ be an extension of $V_{0}$ in $L(x)$ (i.e., $V^{*}$ is a valuation ring of $L(x)$ such that $\left.V^{*} \cap K\left(t_{1}, \ldots, t_{n}\right)=V_{0}\right)$. Since $L(x)$ is algebraic over $K\left(t_{1}, \ldots, t_{n}\right)$, the residue class field $V^{*} / \mathfrak{p}^{*}$ of $V^{*}$ is a finite algebraic extension of $K$. Set $V=V^{*} \cap L$. Then $V$ is of rank $n$ because $t_{i}$ are all in $L$, hence trans. $\operatorname{deg}_{k} V /\left(p^{*} \cap V\right) \leq \operatorname{trans.deg}_{k} L-n<\operatorname{trans.} \operatorname{deg}_{k} L(x)-n=$ trans.deg $\operatorname{deg}_{k} K$. Thus $V^{*} / \mathfrak{p}^{*}$ is not algebraic over $V /\left(\mathfrak{p}^{*} \cap V\right)$, hence is ruled by Theorem 1. Since $V^{*} / p^{*}$ is a finite algebraic extension of $K$, we got a contradiction to that $K$ is anti-rational. Thus Theorem 2 is proved.

Corollary 1. Let $L$ be a finitely generated field over a field $k$. If subfields $K_{1}$ and $K_{2}$ of $L$ are anti-rational over $k\left(k \subseteq K_{i}\right)$, then $K_{1}\left(K_{2}\right)$ is also anti-rational over $k$. Therefore there is the largest anti-rational subfield of $L$ over $k$.

Proof. Assume that $K_{1}\left(K_{2}\right)$ is not anti-rational. Then there is a finite algebraic extension $M$ of $K_{1}\left(K_{2}\right)$ which is ruled over $k: M=M^{\prime}(t), t$ transcendental over $M^{\prime} \supseteq k$. Since $K_{i} \subseteq M^{\prime}(t)$, we have $K_{1}\left(K_{2}\right) \subseteq M^{\prime}$. This contradicts to that $M^{\prime}(t)$ is algebraic over $K_{1}\left(K_{2}\right)$.

Corollary 2. Let $L$ be a field finitely generated over a field $k$. If $L$ is, in two ways, pure transcendental extension of common transcendence degree, say $n$, over its two subfields $K$ and $K^{\prime}$ containing $k$ and if $K$ is anti-rational over $k$, then $K^{\prime}=K$.

Proof. Since $K \subseteq K^{\prime}\left(t_{1}, \ldots, t_{n}\right)=L$ with algebraically independent elements $t_{1}, \ldots, t_{n}$ over $K^{\prime}$, we see that $K \subseteq K^{\prime}$. Since $K$ is algebraically closed in $L$, we have $K=K^{\prime}$.

Theorem 3. Let $L$ be a finitely generated field over a field $k$. Let $\dot{A}$ be the largest anti-rational subfield of $L$ over $k$. Then $L$ is quasi-rational over $A$.

Proof. We prove the assertion by induction on trans. $\operatorname{deg}_{A} L=r$. If $r=0$, 
then $L$ must be anti-rational, and $L=A$. This settle the case. Assume now that $r>0$. Assume that a field $K$ between $A$ and $L$ is anti-rational over $A$. Since $L$ is not anti-rational over $k$ (because of the assumption that $r>0$ ), there is a finite algebraic extension $L^{\prime}$ of $L$ which is ruled over $k: L^{\prime}=L^{\prime \prime}(t), t$ transcendental over $L^{\prime \prime} \supseteq k$. Since $A$ is anti-rational and contained in $L^{\prime}$, we have $A \subseteq L^{\prime \prime}$. Hence $A \subseteq L^{\prime \prime} \cap L$. Since $K$ is anti-rational over $A$, we see that $K \subseteq L^{\prime \prime} \cap L$ similarly. Thus $K$ is an anti-rational subfield of $L^{\prime \prime} \cap L$ over $A$. Hence, by our induction assumption, we have $K=A$. This completes the proof.

\section{Quasi-rational fields}

The following is obvious by the definition of quasi-rationality:

LEMma 3. If a field $L$ is quasi-rational over its subfield $k$, then any field between $k$ and $L$ is quasi-rational over $k$.

Now we prove the following

TheOREM 4. Let $L$ be a finitely generated field over a field $k$. If there is a sequence of fields $L_{i}$ such that $k=L_{0} \subset L_{1} \subset \cdots \subset L_{n}=L$, each $L_{i}(i=1, \ldots, n)$ being quasi-rational over $L_{i-1}$, then $L$ is quasi-rational over $k$.

Proof. It suffices to prove the case $n=2$. We prove the assertion by induction on $r=\operatorname{trans} \cdot \operatorname{deg}_{k} L$. Assume that $K$ is an anti-rational subfield of $L=L_{2}$ over $k$. There is a finite algebraic extension $L^{\prime}$ of $L$ which is ruled over $L_{1}: L^{\prime}=L^{\prime \prime}(t), t$ being transcendental over $L^{\prime \prime} \supseteq L_{1}$. Then $L^{\prime \prime}$ contains $K$. Thus we see that $L^{*}=L^{\prime \prime} \cap L$ has an anti-rational subfield $K$ over $k$. On the other hand, $L^{*}$ is quasi-rational over $L_{1}$ by virtue of Lemma 3. Therefore, by our induction assumption, we see that $L^{*}$ is quasi-rational over $k$. Therefore the anti-rationality of $K$ implies that $K=k$, which proves Theorem 4 .

\section{Zariski problem}

The motivation of the present work arose from the following

ZARISKI PROBLEM.") Let $K$ and $K^{\prime}$ be finitely generated fields over a field $k$. Assume that simple transcendental extensions of $K$ and $K^{\prime}$ are $k$-isomorphic to each other. Does it follow that $K$ and $K^{\prime}$ are $k$-isomorphic to each other?

7) This problem was raised by O. Zariski in 1949 at Paris Colloquium on algebra and the theory of numbers. Cf. [4]. 
In other words, this asks whether or not the condition $K(x)=K^{\prime}\left(x^{\prime}\right)$, with $x, x^{\prime}$ transcendental over $K, K^{\prime}$ respectively, implies $K \cong K^{\prime}$.

This problem is not solved yet. But there are some special cases where the answer is affirmative.

One important remark is that $k$ may be replaced by an arbitrary anti-rational subfield of $K(x)$. This remark gives easy proof in each of the following cases.

(i) $K$ is anti-rational over $k$. In this case, $K=K^{\prime}$.

(ii) $K$ is a separably generated extension of $k$ and trans.deg $\operatorname{deg}_{k} K=1$.

(iii) $k$ is algebraically closed, trans.deg $k=2$, and $K$ contains an antirational subfield $A$ over $k$ which is different from $k$.

(iv) $k$ is an algebraically closed field of characteristic zero and trans. $\operatorname{deg}_{k} K=2{ }^{8 \text { ) }}$

Another easy affirmative case is:

(v) $x=x^{\prime}$ and $k$ has infinitely many elements. ${ }^{9}$

\section{Some other problems}

For simplicity, let $k$ be an algebraically closed field. Then for function fields over $k$, there are obvious implications:

$$
\text { Rational } \Rightarrow \text { uni-rational } \Longrightarrow \text { quasi-rational. }
$$

Existence problem of uni-rational fields seems to the writer to be still open, though the existence has been claimed by Italian geometers in three dimensional, characteristic zero case. (In positive characteristic case, Zariski gave an example of a uni-rational field of transcendence degree 2 which is a purely inseparable extension of a rational field. The open problem is the existence of uni-rational fields whose separable extension can be rational.) We like to add here the existence problem of quasi-rational fields. In particular:

Let $t_{1}, \ldots, t_{n}$ be algebraically independent elements over $k$ and let $f_{1}, f_{2}, f_{3}$ be non-zero elements of $k\left[t_{1}, \ldots, t_{n}\right]$. Let $K$ be the field $k\left(t_{1}, \ldots, t_{n}, x, y\right)$ with defining equation $f_{1} x^{2}+f_{2} y^{2}=f_{3}$. Then $K$ is quasi-rational over $k$. Are there any choice of $n, f_{1}, f_{2}, f_{3}$ such that $K$ is not uni-rational? ${ }^{10)}$

There is another problem:

\footnotetext{
8) This case was discussed by Segre [4].

9) See footnote 5 ).

30: If $n=1$, then $k\left(t_{1}\right)$ is a $C_{1}$-field, hence $K$ must be rational.
} 
Is Theorem 1 true without assuming the condition (ii)?

As for this, we can prove the following

Theorem 5. Let $K, K(x), V^{*}, p^{*}, V$ and $\mathfrak{p}$ be as in Theorem 1, but not assuming (ii). Then $V^{*} / p^{*}$ is a subfield of a simple transcendental extension of the algebraic closure of $V / \mathfrak{p}$.

Proof. In order to prove this, we may assume that $K$ is algebraically closed. Let $y \in V^{*}$ be such that $y$ modulo $\mathfrak{p}^{*}$ is transcendental over $V / \mathfrak{p}$. Then $V^{*}$ contains $V(y)=V[y]_{p v[y]}$. Since $K(x)$ is a finite algebraic extension of $K(y)$, we see that the value group $G$ of $V$ is of finite index in that of $V^{*}$. Since $K$ is algebraically closed, $G$ is divisible, hence the value group of $V^{*}$ coincides with $G$. $y$ can be expressed in the form $\Pi\left(a_{i} x-b_{i}\right) / \Pi\left(c_{j} x-d_{j}\right)\left(a_{i}, b_{i}, c_{j}, d_{j} \in K\right)$. Since $G$ coincides with the value group of $V^{*}$, we may assume that these linear factors are units in $V^{*}$. Since $y$ modulo $\mathfrak{p}^{*}$ is transcendental over $V / \mathfrak{p}$, we see that at least one of these linear factors is transcendental modulo $p^{*}$ over $V / p$. Thus we may assume that $y$ is a linear polynomial in $x$. Then $K(x)=K(y)$ and $V^{*}=V(y)$. Hence $V^{*} / p^{*}=(V / p)\left(y\right.$ modulo $\left.p^{*}\right)$, which completes the proof.

\section{REFERENCES}

[1] S. Abhyankar, On the valuations centered in a local domain, Amer. J. Math. 78 (1956), 321-348.

[2] J. Igusa, On a theorem of Lüroth, Mem. Univ. Kyoto 26 (1950-51), 251-253.

[3] P. Samuel, Some remarks on Lüroth's theorem, Mem. Univ. Kyoto 27 (1952-53), 223-224.

[4] B. Segre, Sur un problème de M. Zariski, Colloque internat. d'algèbre et de théorie de nombres, Paris 1949, 135-138.

[5] H. Weber, Lehrbuch der Algebra, II, Braunschweig, 1899.

\section{Harvard University}

and

Kyoto University 